\title{
Virtual Sightseeing in Immersive 3D Visualization Lab
}

\author{
Jacek Lebiedź, Mariusz Szwoch \\ Gdańsk University of Technology \\ Faculty of Electronics, Telecommunications and Informatics \\ Department of Intelligent Interactive Systems \\ G. Narutowicza St. 11/12, 80-233 Gdańsk, Poland \\ Email: \{jacekl, szwoch\}@eti.pg.gda.pl
}

\begin{abstract}
This paper describes the modern Immersive 3D Visualization Lab (I3DVL) established at the Faculty of Electronics, Telecommunications and Informatics of the Gdańsk University of Technology (GUT) and its potential to prepare virtual tours and architectural visualizations on the example of the application allowing a virtual walk through the Coal Market in Gdańsk. The paper presents devices of this laboratory (CAVE, walk simulator etc.), describes methods of "immersing" a human in a virtual environment (city, building etc.) and discusses future possibilities for development (directions of research and limitations of today's hardware and software).
\end{abstract}

\section{INTRODUCTION}

$\mathrm{V}$ IRTUAL reality (VR) is over 50 year old, now. The first devices like virtual reality video arcade Sensorama Simulator or stereoscopic-television apparatus for individual use (HMD Head-Mounted Display) were invented in the 1960s [1]. Initially, applications of VR devices were very restricted due to their high price and technological limitations, but now, fifty years later, such devices become very popular on the customer market (e.g. Oculus Rift, HTC Vive). Their relatively low price, acceptable reliability and passable level of immersion (despite screen-door effect) allow us to use them commonly for video games and other kinds of virtual reality experience.

The virtual reality CAVE is much younger than the HMD. The first Cave Automatic Virtual Environment (CAVE) came into being in the early nineties at the University of Illinois $[1,2]$. Generally, the CAVE may be defined as a cuboidal chamber that has stereoscopic projection screens instead of the walls, the floor, and sometimes the ceiling. A human visitor, wearing only lightweight $3 \mathrm{D}$ glasses, is surrounded by a $3 \mathrm{D}$ virtual scene projected by projectors placed outside the CAVE. The three-dimensional impression is intensified by additional adjustment of the images forming the scene to the location of a human head. Visual effects are often supported by a 3D surround audio system. Therefore, the level of immersion is very high for CAVEs.

Dozens of CAVEs have been constructed within the last twenty five years $[3,5]$. Some of them are rather simple and

This work was supported in part by DS Funds of the Faculty of ETI of the Gdańsk University of Technology. consist of only four screens, usually three walls and a floor (e.g. the first CAVE in Poland [6]). Other CAVEs are more sophisticated and contain more screens [12]. Four walls indicate that one of them has to be a gate. The complete sixfaced cuboidal CAVEs with four walls, a floor and a ceiling are rather rare.

\section{IMMERSIVE 3D VISUALIZATION LAB}

The Immersive 3D Visualization Lab [7, 8, 9, 10] contains complete six-faced cubical CAVE made of thick square acrylic plates (Fig. 1). A spectator can see a 3D scene on each CAVE's face using a $120 \mathrm{~Hz}$ stereoscopy system in passive mode (spectrum channels separation by selective interference filters) or active one (separation in time with active shutter glasses [10]). The viewer's glasses have special markers that are tracked by four infrared cameras placed in the upper corners of the CAVE. Eight speakers located in the same corners and a subwoofer standing outside the CAVE provide surround audio system.

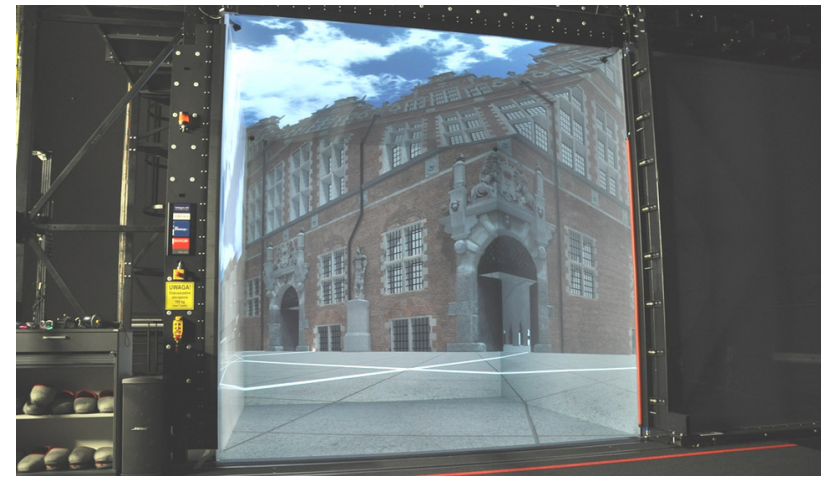

Fig. 1 The CAVE in the I3DVL (the gate of the CAVE is open)

Simulation participants may walk freely in the CAVE from wall to wall, as in any typical CAVE. However, unlimited virtual wandering is also possible using a handheld controller, called wand or fly-stick. Unlike other common solutions, the CAVE in the I3DVL can use a spherical walk simulator as an additional movement controller (Fig. 2). The spherical walk simulator $[4,11,13]$ has a form of an openwork sphere, that freely rotates on rollers with a small friction. One can treat it like a human size omnidirectional "hamster wheel". A user may walk 
inside the sphere watching, through its rotating surface, the images on the CAVE screens that change according to the direction and speed of the sphere revolutions. This solution allows the user to march on foot through the virtual world projected on the CAVE screens without any space limitations. There are no other CAVEs in the world with such possibility.

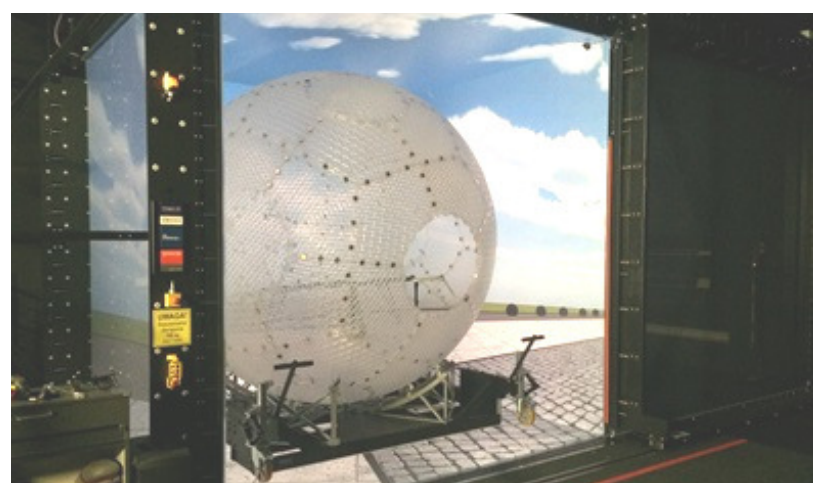

Fig. 2 The spherical walk simulator inside the CAVE in the I3DVL (the gate of the CAVE is open)

The CAVE in the I3DVL can be used with the spherical walk simulator, but both devices can also work separately: the CAVE in the typical configuration with a handheld controller and the spherical walk simulator with the HMD. The specially designed and constructed transport trolley allows to roll the spherical walk simulator in and out (Fig. 2) without a risk of collision with the CAVE's screens. The diameter of the spherical walk simulator is $3.05 \mathrm{~m}$, and the edge of the cubical CAVE is $3.4 \mathrm{~m}$ long. Hence, there is only about $17 \mathrm{~cm}$ between the surface of the $250 \mathrm{~kg}$ sphere and the wall of the CAVE from each side.

Every wall-screen obtains two images from two WUXGA $(1920 \times 1200)$ projectors. The final image, combined by blending, has a resolution $1920 \times 1920$. Thus, all six screens require twelve projectors driven by dozen computers. Two additional computers control virtual scene dynamics, real object tracking, sound generation, and video surveillance.

\section{SOFTWARE PLATFORMS}

The Immersive 3D Visualization Lab can work in various software environments that support multicomputer (cluster) process synchronization. Each computer can operate under control of Windows or Linux operating systems, and VR programmers may use both of them. At present, the most popular software platform in the I3DVL is Unity - the environment dedicated to video game developers. Low-level graphics programming in $\mathrm{C}++/ \mathrm{C} \#$ and OpenGL or Direct $\mathrm{X}$ is also used, particularly for experiments with global illumination rendering methods like raytracing and radiosity. Use of other tools for game development (e.g. Unreal Engine) or virtual reality applications (e.g. Bohemia VBS3, Presagis Vega Prime) is possible, too.

The computational power of I3DVL computers might not be sufficient for some complex tasks, such as sophisticated rendering, nontrivial physics calculations, advanced artificial intelligence or prediction of user behavior. In such cases, in order to provide efficient real-time processing, the power of high performance cluster Tryton from the Academic Computer Center in Gdańsk (CI TASK), connected via the fast optical fiber InfiniBand, can be used [14]. This connection allows treating the computers of the I3DVL and the nodes of the cluster Tryton as one uniform cluster.

\section{The COAL MARKET VIRTUAL SIGHTSEEING}

The Coal Market is one of the historical squares of Gdańsk with many landmarked buildings, e.g. the Great Armoury (Fig. 3), the Straw Tower, the Court of the Brothership of St. George (Fig. 4), the Torture House, the Prison Tower, the Golden Gate (Fig. 4), the Upland Gate, and several cultural institutions. Regrettably, the contemporary buildings at the Coal Market's western frontage contrast with the historic and cultural character of the Market. Therefore, the City Council of Gdańsk announced a competition for new urban-architectural concepts of this square. Over 10 teams from Faculty of Architecture at GUT took part in this contest and, finally, three architectural projects were awarded (Fig. 5-7).

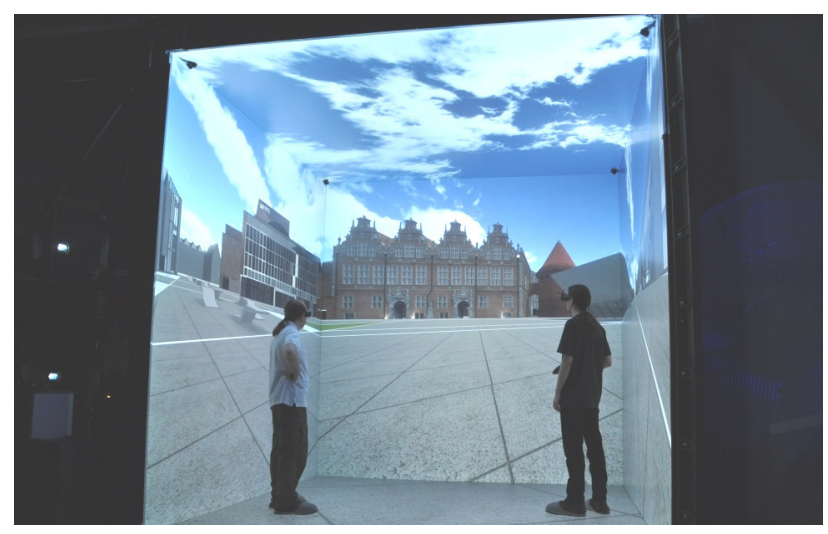

Fig. 3 The virtual Great Armoury

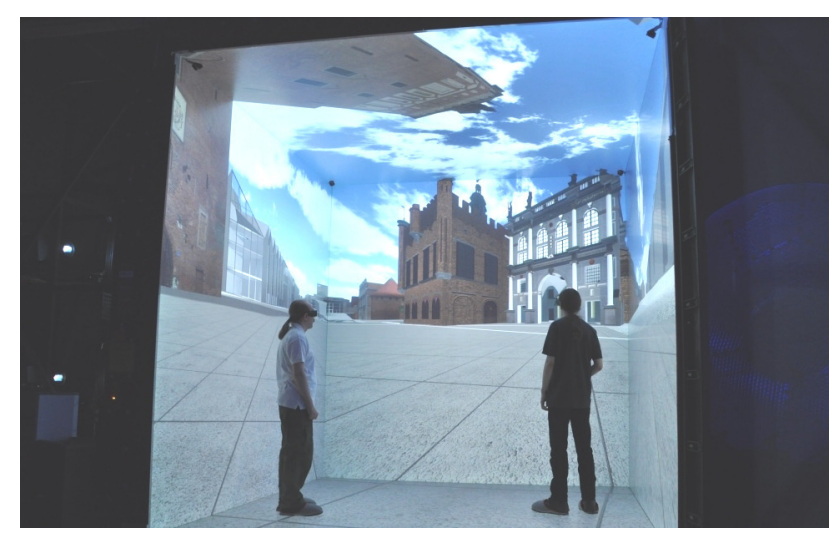

Fig. 4 The Court of the Brothership of St. George and the Golden Gate

This contest provided a good opportunity to propose a new approach to visualize the awarded projects not only as a limited set of static architectonic sketches but as dynamic, interactive real time visualization in the CAVE environment. Spectators, e.g. architects or council members, could observe the modeled urban area from practically any 


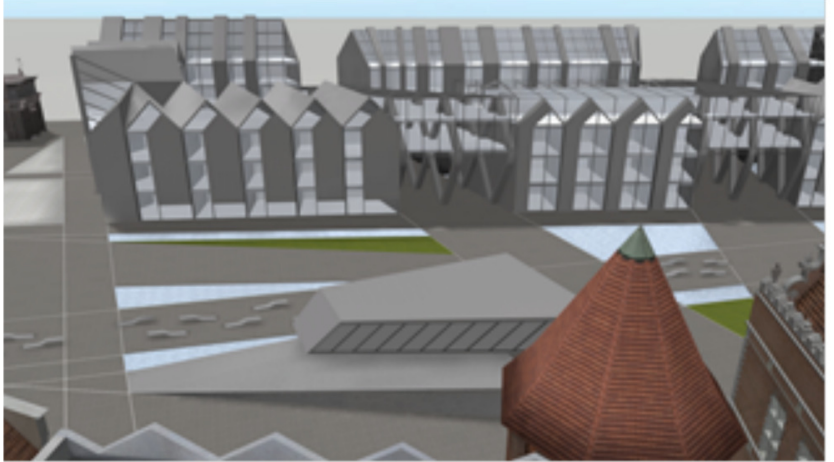

Fig. 5 Visualization of the western frontage of the Coal Market (project by E. Kowalik et al.)

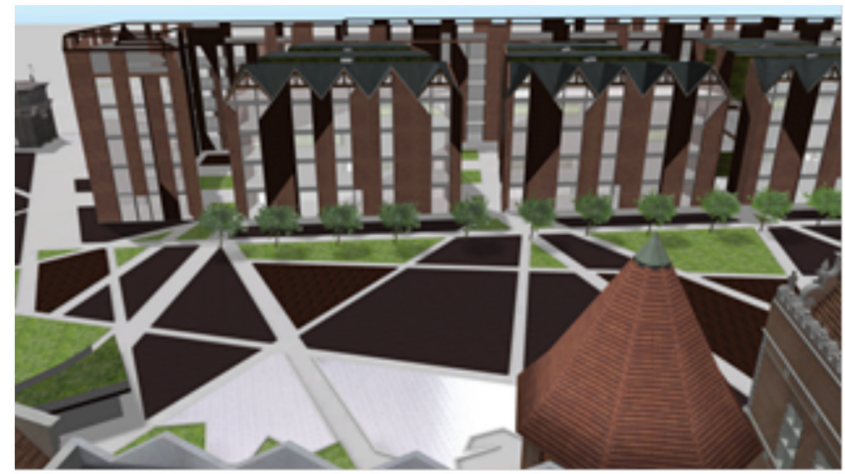

Fig. 6 Visualization of the western frontage of the Coal Market (project by M. Chrzanowska et al.)

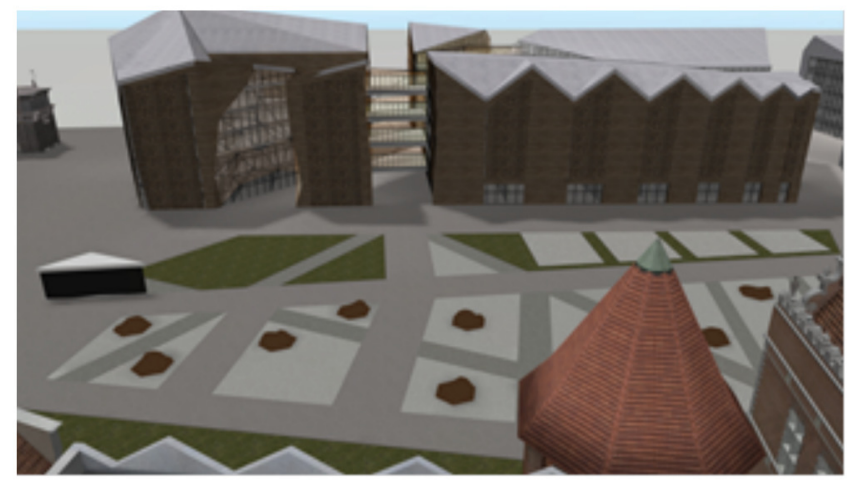

Fig. 7 Visualization of the western frontage of the Coal Market (project by $\mathbf{L}$. Plata et al.)

viewpoint, and freely walk along virtual houses and buildings with true $360^{\circ} 3 \mathrm{D}$ surrounding view.

Thus, the goal of the project described in this paper was to fully model the Coal Market urban area with three different variants of its western frontage buildings. Additionally, the requirements specification assumed automatic and free walk functionality, and capability of switching between several predefined viewpoints and frontage versions.

Although all models from competition were available in electronic form, two severe reasons prevented their direct usage in the CAVE visualization. Firstly, all adjacent buildings were modelled as single, large 3D objects. While such approach may be used for not real-time architectural visualizations, it prohibits the use of any optimization algorithms in modern 3D graphics engines, such as Level of Detail (LOD) techniques, occlusion culling, and other. Secondly, visualization of all unchanged historical buildings was based on very simplified low-poly models with no textures nor normal vectors but with some geometric errors instead. Although these imperfections were hardly noticeable in static architectural visualization, where these buildings provided the background for contest models, they were unacceptable for interactive CAVE visualization, where all buildings at the Coal Market were equally important.

For these reasons the project's decision was made to model all the Coal Market's buildings from scratch as separate 3D objects. Such models should have significantly reduced complexity while still preserving visualization quality. According to these assumptions, two architect students created new 3D models both for three contest propositions, as well as for ten buildings located in other untouched frontages of the square.

Based on provided models three 3D scenes of the Coal Market were designed in Unity environment with three different variants of the square's western frontage (Fig. 5-7). Unfortunately, the models prepared by the architect students were too detailed, with hundreds of thousands of vertices, even for not significant architectonic details, such as ornate wrought-iron gate's lattice (Fig. 8), dome-like roof details or roof sculptures. Initially, the whole scene consisted of over 5 million vertices, which occurred too complex for real-time visualization, resulting in low refresh ratio lower than 10 fps. Thus, most models had to be simplified by dramatic reduction of the number of their vertices with minimal loss of quality. This reduction was performed by an IT student with 3D modelling skills. Sample effects of performed complexity reduction are presented in Fig. 8. The original 3D model of wrought-iron lattice (a) consisted of nearly 670 thousand vertices and 1.2 million triangles, while the simplified one (b) is built from only 25 vertices and one partially-transparent mid-resolution texture. Original high complexity of the model (c) is not visible during standard CAVE visualization (d).

Finally, the scene complexity were reduced by a factor of 10 resulting in about 500000 vertices in total. This reduction, accompanied by well-known optimization techniques such as LOD, occlusion culling and light mapping, allowed for effective real-time scene visualization in CAVE environment at refresh rate of about 120 fps.

Additional feedback from architects pointed the way of visualization's improvement by adding simplified building models that would surround the modelled Coal Market and provide some visual background both for a bird's-eye view of the square as well as for wandering around its neighborhood. Such buildings were added based on the simplified building models consisted of only 10 vertices each, which did not increase the overall scene complexity in practice. The results are presented in Fig. 9 where additional buildings are visible in the background. 
a)

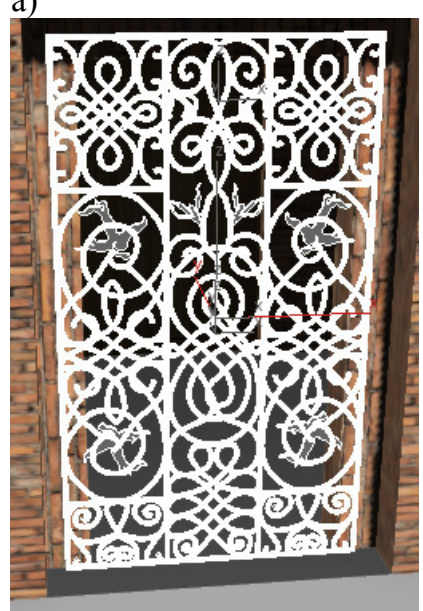

c)

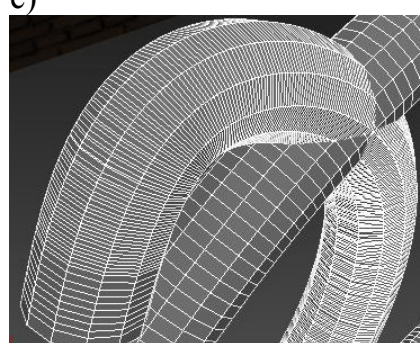

b)

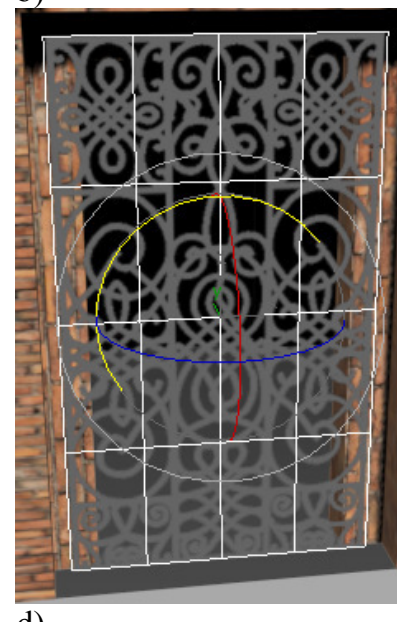

d)

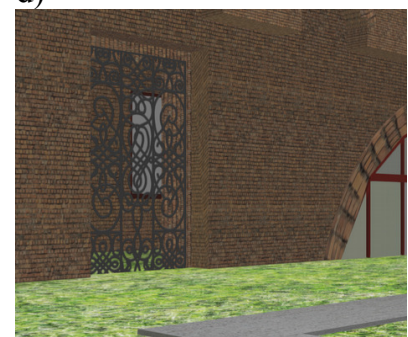

Fig. 8 Sample model of wrought-iron gate's lattice: a) highly detailed original (white color is used for vertices and edges), b) simplified flat model, c) magnified detail of the original model, d) final appearance in $3 \mathrm{D}$ visualization

\section{VIRTUAL SIGHTSEEING}

Visualization of the Coal Market became the first application demonstrating I3DVL capabilities. It was presented to many visitors who confirmed high usability of the CAVE environment in the field of architectural visualizations. Possibility of almost free interaction within a 3D scene and high immersion level, which is guaranteed by the surrounding view, allow for better scene perception. For example, most interaction participants enjoyed the possibility of visiting every nook and cranny of the buildings and courtyards. This possibility of visual testing of all inner passages and communication courses occurred as vital as the general view of building frontage.

Also, the possibility of immediate switching between different scene versions makes their comparison easier than at traditional exhibition as it gives the same visual context. Additional application feature allows for real-time switching between daylight and night scenes (Fig. 10, 11), and thereby the visitors can verify how the place would look like by night with artificial illumination. Yet another kind of experience is the possibility of bird's eye view from highly located viewpoints (Fig. 5-7,9) which is especially realistic inside the CAVE.

A wide multitude of presentations for different peoples have allowed for many interesting observations. For example, some visitors have serious problems with movement control in the CAVE. For such persons the

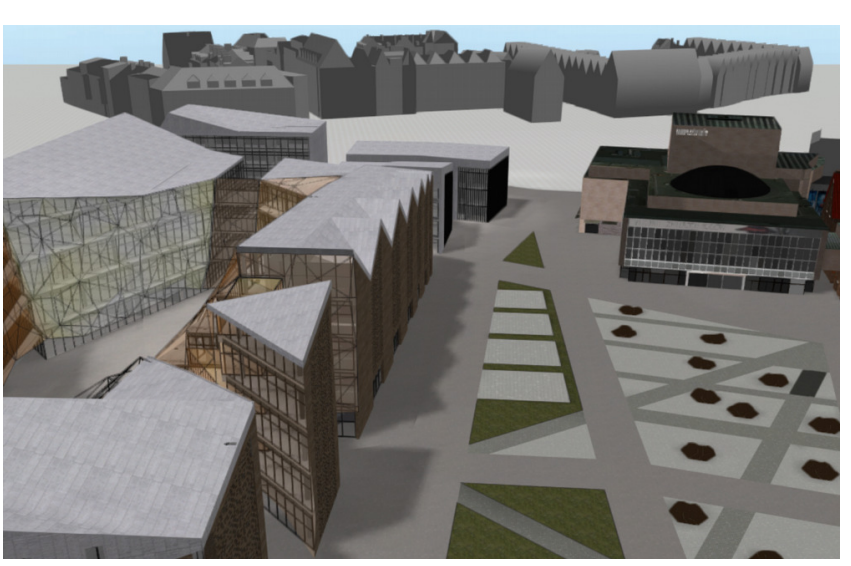

Fig. 9 The bird's-eye view of the virtual Coal Market with simplified buildings in the background

automatic walk mode occurred a real salvation, allowing for simple looking around while being moved forward along the predefined path as inside a sightseeing trolleys or bus. It was also confirmed that some people do have some problems with 3D image perception using 3D glasses. Fortunately, most of them reported that the problem is far less than with the HMDs such as Oculus Rift. This surely results from much higher image quality as well as from better user orientation inside the CAVE. Moreover, a man inside the CAVE can see his own body and other participants of the simulation. The depth of immersion in the CAVE over the HMD seems to be unquestionable, but conclusive proving this general thesis requires further research comparing the reactions of people using different VR devices and various scenarios by means of questionnaires, behavioral observation and biological measurements (as heart rate, blood pressure and electrical activity of the brain).

The project realization allows also for gathering invaluable experience and clues for further application development for I3DVL's CAVE environment. For example, many inbuilt Unity mechanisms, such as scene switching or interface elements, had to be redesigned due to the clientserver architecture of the CAVE and the need of the network synchronization between I3DVL's computers. Moreover, such a presentation of $3 \mathrm{D}$ image requires higher rendering rate than for a $\mathrm{PC}$ demonstration.

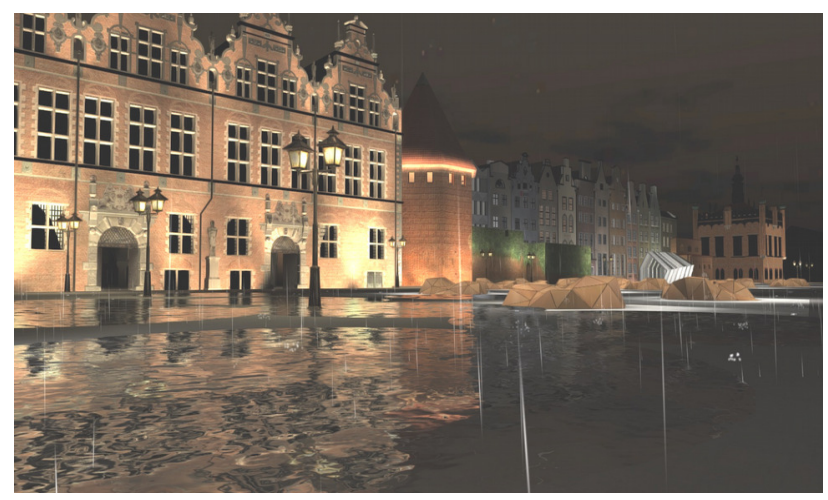

Fig. 10 A night scene of the Coal Market with rain simulation 


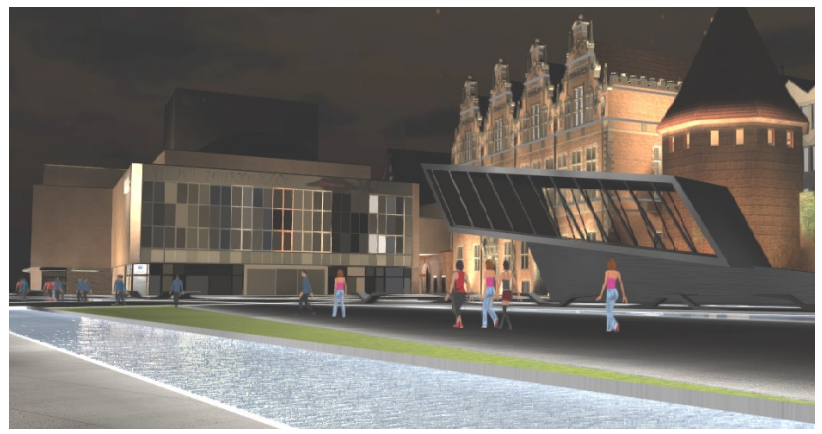

Fig. 11 A night scene of the Coal Market with crowd simulation

\section{CONCLUSION AND FUTURE WORK}

Virtual sightseeing can be useful not only for virtual tourism, but also for virtual prototyping, allowing assessment of designed objects, like urban areas, squares, streets, buildings with their interiors, and even crafts, vehicles, machines etc. Believable visualization can help in decisionmaking without the use of spatial imagination. Just take a stroll through the virtual scene and decide.

Future works are focused on using of additional computational power, provided by the high performance Tryton cluster at CI TASK, to expand the Coal Market scene with crowd, snow and rain simulations. The first experiments with developed scalable particle system for cluster-aided visualization proved successful in weather phenomena and crowd simulation (Fig. 10,11), though many problems of latency-critical processing have to be solved, yet.

\section{ACKNOWLEDGMENT}

Authors would like to thank Małgorzata Chrzanowska and Lukasz Plata for preparing 3D architectural models, and Adrianna Szwoch for 3D scene modelling and scripting.

\section{REFERENCES}

[1] G. Burdea, P. Coiffet: Virtual Reality Technology, 2nd Ed., Wiley, New York 2003.

[2] C. Cruz-Neira, D. J. Sandin, T. A. DeFanti: The CAVE: A Virtual Reality Theater. HPCCV Publications, 2, 1992.

[3] T. A. De Fanti et al.: The future of the CAVE. Central European Journal of Engineering. November 2010.

[4] J. Gantenberg, K. Schill, C. Zetzsche: Exploring Virtual Worlds in a Computerised Hamster Wheel. German Research 1/2012.

[5] Iowa State University News Service: The most realistic virtual reality room in the world. 2006.

[6] J. Jurkojé, P. Wodarski, R. Michnik, M. Gzik, A. Bieniek: The influence of visual parameters of a scenery on the ability to keep the balance in the virtual reality. 13th International Symposium on $3 D$ Analysis of Human Movement, Lausanne 2014, pp. 88-91.

[7] J. Lebiedź, J. Lubiński, A. Mazikowski: An Immersive 3D Visualization Laboratory Concept. Proc. of the 2nd Int. Conf. on Information Technology - Information Technologies, Vol. 18, 2010, pp. $117-120$.

[8] J. Lebiedź, A. Mazikowski: Launch of the Immersive 3D Visualization Laboratory. Szybkobieżne Pojazdy Gasienicowe, vol. 34, nr 1, 2014, pp. 49-56.

[9] J. Lebiedź, A. Mazikowski: Innovative Solutions for Immersive 3D Visualization Laboratory. 22nd International Conference on Computer Graphics, Visualization and Computer Vision WSCG 2014 Communication Papers Proceedings (ed. Vaclav Skala), Plzeň 2014, pp. 315-319.

[10] A. Mazikowski, J. Lebiedź: Image projection in Immersive 3D Visualization Laboratory. 18th International Conference in Knowledge Based and Intelligent Information and Engineering Systems KES 2014, Gdynia 2014, Procedia Computer Science 35, 2014, pp. 842-850.

[11] E. Medina, R. Fruland, S. Weghorst: Virtusphere: Walking in a Human Size VR Hamster Ball, Proc. of the Human Factors and Ergonomics Society Annual Meeting 52, №. 27, 2008, pp. 2102-2106.

[12] A. Mitchell: Lost in 1-Space. INAVATE IML, 10 2012, pp. 3-4. http://www.inAVateonthenet.net .

[13] VirtuSphere Inc.: Virtusphere. The Virtual World Immence. Let's Immerse Together. http://www.virtusphere.com/index.html .

[14] Ł. Wiszniewski and T. Ziółkowski: Real-Time Connection between Immerse 3D Visualization Laboratory and KASKADA platform. TASK Quarterly 19, No 4, 2015, pp. 471-480. 